\title{
Measuring Time
}

\author{
Sydney Baldwin Self \\ United States \\ sydself@alum.mit.edu
}

\section{Abstract}

I. The speed of light is an absolute, the measurement of the speed of light is not an absolute.

II. According to Special Relativity, the rate at which a clock ticks is a function of the speed at which it is travelling through space; the faster the speed, the slower the rate. This is called time dilation.

III. Time dilation is a function of the speed of an object through space. The faster an object is travelling the greater the time dilation.

IV. Because of time dilation, we are unable to measure the absolute speed of an object through space.

V. We do not actually measure time. Instead we have instruments which measure the physical characteristics of instruments which are affected by time. We then interpret these physical characteristics and this interpretation becomes our measurement of time.

VI. Our body is one of the instruments we use to measure time, but we also use various clocks.

VII. Time is not a characteristic of the universe; it is a construct which can be defined to have any value we choose.

VIII. If we could establish a stationary point in space, it would be possible to:

a. measure the absolute speed of light.

b. measure the absolute speed through space of any moving object.

c. establish a clock for absolute time.

IX. It might be theoretically possible to establish such a point, as follows:

a. Assume a space station, located at a Lagrange point.

b. Modify the speed of the space station through space for the $x, y$ and $z$ axes to maximize the speed of light.

X. The location of the space station when the speed of light was maximized would be at a stationary point in space.

\section{Keywords}

Speed of Light

Planck Time

Time Dilation

Hafele and Keating

Stationary point in space 
Academic Discipline - Physics

Sub-Discipline - Time

Approach - Logical Analysis

\section{Introduction}

In 1887 scientists believed that just as sound was transmitted using air as a medium, light was transmitted through a medium which was known as the 'stationary luminiferous æther'. The MichelsonMorley experiment ${ }^{1}$ was intended to detect the motion of light through the æther; instead it provided evidence that the æther did not exist.

In 1905 Einstein published a paper that has become known as Special Relativity ${ }^{2}$ which was derived from the results of the Michelson-Morley experiment.

\section{Special Relativity}

Special Relativity is based on two postulates:

1. The laws of physics are invariant in all inertial systems.

2. The speed of light in a vacuum is the same for all observers regardless of the motion of the light source.

In other words, Einstein recognized that the speed of light is a universal, absolute constant. This concept may well be the most important scientific fact that has ever been discovered. It is hard to discuss any topic dealing with physics that doesn't, somewhere, refer to that fact, either directly or indirectly.

It is important to understand that postulate \#2 defines the 'speed of light' as an absolute constant; it should not be inferred that postulate \#2 applies to the observed speed of anything other than the speed of light. Also, postulate \#2 references 'observers' but in my article 'The Nature of Time - a $21^{\text {st }}$ Century View"', I show that the laws that govern the nature of the universe must have existed before there were observers, therefore the phrase "for all observers" is not relevant. It could and should be omitted from postulate \#2.

Special Relativity introduced a new concept, namely that of time dilation $(\gamma)$. When an object moves through space, the rate at which it experiences time is a function of the speed at which it is moving through space; the faster the object is moving through space, the more slowly it experiences time. Since Earth is moving through space, time passes more slowly for objects on earth than it would if Earth were stationary in space.

An illustrative example is that according to Special Relativity the absolute value of speed of light on Mars is the same as the absolute value of the speed of light on Earth, but the measured value of the speed of light on Mars is not the same as the measured value of the speed of light on Earth because the two planets move through space at different speeds.

Exactly what is time dilation? The simplest way to define time dilation is to refer to the Lorentz transformation, namely

$$
\gamma=\frac{1}{\sqrt{1-\frac{v^{2}}{c^{2}}}}
$$

In this equation ' $c$ ' equals the speed of light and ' $v$ ' equals the velocity of the object whose speed is being measured. (In my paper "Measuring Velocity" ${ }^{4}$ I question the mathematical validity of dividing a vector 
term by a scalar term. I suggest that we could substitute ' $V$ ' for ' $V$ ' and define ' $V$ ' as the speed of the object not its velocity).

As can be seen from the above equation, if the speed of an object is equal to the speed of light, the value of the associated time dilation is infinite; if an object is stationary in space (if such an object exists) the value of time-dilation is zero. (I find it easier to understand time dilation if one inverts it and calls it aging).

Light is composed of photons and photons have two interesting characteristics:

1. Photons do not experience time, so photons do not experience aging; this is confirmed by the Lorentz transformation. This is a very important, under recognized fact, and it is why Postulate \#2 above only applies to the speed of light.

2. Photons always travel at the same speed; in fact stationary photons do not exist. Although photons do not experience time dilation, any device that is used to measure the speed of a photon has to experience time dilation.

In my article "A logical examination of the nature of time" ${ }^{5}$ I propose that when absolute time ticks (again see my article about 'A $21^{\text {st }}$ Century View ${ }^{3}$ ) a particle may move a Planck length through space or experience a Planck time through time, but it may not do both.

Which it does is based on probability - defined as the speed of the object divided by the speed of light.

This proposal, when applied in conjunction with the Lorentz transformation, gives a logical explanation for time dilation.

\section{Measuring Time}

The Universe has three components - matter/energy, space and time; each has its own set of characteristics. Matter/energy exists physically and has physical characteristics that can be measured — for example the length and weight of objects are what they are, and exist whether or not they are observed. We assign units of measure to these characteristics but the units used to measure matter are constructs, not characteristics of the objects - we can measure a length in feet or in meters but it doesn't change the physical length of the object.

Matter/energy is defined by Quantum Mechanics and is not discussed further in this article.

As far as space is concerned, we can't even define its characteristics, let alone measure them.

To measure time, first we have to understand what time is. To begin with, we don't really measure time, what we measure is the effect that time has on the instruments used to measure it.

One of these 'instruments' is the human body: the passage of time results in changes to the human body - we get older, our whole body grows as do our finger nails and our hair, women become pregnant and the duration of their pregnancy is a measurement of time - in other words, as living creatures we experience time and we use our experience as a base (but not the only base) for measuring the duration of time.

We also use instruments and tools to measure time. These instruments and tools are very different from those we use to measure matter because we don't use them directly; instead we have to interpret the measurements provided by the instrument and the interpreted value is the measurement of time.

For example, it is probable that the first measurement of time was the sundial. The sundial is composed of a vertical stick which casts a shadow on a flat surface. A clever person conceived the idea of drawing a scale on the surface with markings which could be used to represent the time of day and its relation to the length of the year; we can relate days and years to our personal experiences. 
The shadow itself was not a characteristic of time, it had no meaning until it was interpreted using the markings on the surface. I doubt if the original markings defined hours, or days; they were added later, probably much later, and they became what could be considered to be the first clock; but physically, the markings were what was used to measure time.

A second way of measuring time is to use a pendulum. A pendulum is also a very simple device; a weight at the end of a wire swings back and forth. It didn't become a device for measuring time until Galileo Galilei observed that the duration of the swing back and forth was not related to the weight of the object nor to the length of the arc of the swing; instead it was based on the length of wire which holds the weight. Galilei had discovered that the duration of the swing can be varied by changing the length of the wire.

The measurement of this duration became a measurement of time, thus the time measured by a pendulum is based on the physical characteristics of a swinging pendulum; it is not a characteristic of time. It required the cleverness of Galilei to realize that this characteristic of a swinging weight could be used to measure time in a form to which we could relate.

I do not know who it was that sub-divided the day into hours, the hours into minutes and the minutes into seconds - perhaps it was Christian Huygens who developed the first pendulum clock in 1656 — but hours, minutes and seconds are all constructs which we associate with the passage of time; they are not physical objects in the sense that the meter which is used to measure distance is a physical object.

A third, more recent, way of measuring time is based on an observable physical characteristic of the cæsium atom which occurs at very regular predictable intervals. Scientists count these intervals and have determined that a specified number of these intervals provide a very accurate measurement of time. Again, we make use of a physical characteristic of matter to provide us with a meaningful way of measuring time; we are not actually measuring time itself.

\section{Measuring the Speed of Light}

Max Planck defined the speed of light as equal to a Planck length divided by a Planck time. He defined a Planck length as the shortest length that could be measured - which has to be an absolute constant - so a Planck time also must be an absolute constant.

The Michelson-Morley experiment provided a very accurate measurement of the speed of light. Since light is composed of photons, measurement of the speed of light is computed by dividing the distance a photon moves by the time it takes for the photon to move that distance. Distance can be measured physically by using a physical measuring stick but measuring time is more complex than measuring distance because, as discussed below, although the actual speed of a photon is a constant, any device that is used to measure the speed of light is subject to time dilation.

\section{Measuring the Speed of Objects}

As discussed above, the equation for measuring the speed of an object is "Speed = Distance $\div$ Time". A Planck length is defined in meters. The length of a meter is not as easy to define as it once was; it used to consist of the distance between two marks on a platinum-iridium stick located at the International Bureau of Weights and Measures in Sevres, France. It now consists of the "length of the path travelled by light in vacuum during a time interval of $1 / 299,792,458$ of a second".

A Planck time is measured in seconds. Defining the duration of the second is not simple. The second used to be $1 / 86,400$ of the length of a day. Then it was discovered that the length of a day was gradually increasing (as the result of the rotational speed of the Earth gradually decreasing), so it was decided to use a different standard; namely "the duration of $919,263,1770$ periods of the radiation corresponding to the transition between the two hyperfine levels of the ground state of the cæsium133 atom." 
It is an interesting, and important, point that time - as we think of it - is not really a characteristic of the universe; it is a construct. If Earth had had a different rotational speed, the duration of the second would be different. We could redefine the second to have any value we chose and, as long as we used it consistently we could do so without re-interpreting our understanding of the universe. On the other hand, the speed of a photon through space is not a construct; it is an absolute. When we say that a second consists of a specified number of ticks of Planck time we are creating a construct.

So, to measure the speed of light, we establish a measured distance and, using a measuring device such as a cæsium clock, we determine the time it takes for a photon to travel that distance. The techniques of doing this are not simple but the principle is.

In 1971 Hafele and Keating ${ }^{6}$ flew cæsium clocks around the Earth in commercial airliners. As a result, they were able to compare the elapsed time of the clocks on the airliners against that of a clock that remained at the U.S. Naval Observatory. One of the clocks flew east, the other flew west. Both clocks were expected to experience time dilation, but the flying clock that flew east was expected to age less than the clock flying west because it was moving faster through space. Also, both flying clocks were expected to age more quickly than the stationary clock because the force of gravity on them was less. The experiment proved that the expectations were correct.

Furthermore, clocks at the equator of the Earth experience time at a slower rate than clocks at the north pole because their speed through space is greater; clocks in the summer experience time at a different rate than clocks in the winter because the Earth travels around the sun in an ellipse, not in a circle; clocks on the space station and on the various GPS satellites experience time at a slower rate than the clocks on Earth and clocks on Mars experience time at a different rate than clocks on Earth.

To summarize, because of time dilation we are unable to accurately determine the exact absolute speed of an object within the frame of reference of Earth; we are unable to determine:

- the speed of Earth through the solar system.

- The speed of our solar system through our galaxy.

- The speed of our galaxy through the universe.

It is perfectly true that the differences in measurement of clocks cited above are very small, but for certain applications, such as GPS systems, today's technology requires measurements that are extremely precise.

\section{Locating a Stationary Point in Space}

Suppose that one could locate a location in space which was not moving! Since an object at such a location would not experience time dilation, it would have a number of uses. For example:

1. The reading of a clock at that location would be a reading of absolute time which could then be used as a standard for Greenwich Mean Time.

2. We could calculate the absolute speed of Earth and other objects in the Solar System.

3. We could establish the absolute speed of other observable objects.

It is a well-known fact that we can only measure the speed of an object as it relates to the speed of another object - there are no reference points in space. But that statement does not prove that a stationary object in space couldn't exist; the problem is in establishing an object in space that is stationary - a far as we know, there is no natural law that prohibits the existence of such an object. 


\section{theoretically.}

Could it be possible to establish a stationary point in space? I think that there is, at least

Let us start with two known facts:

1. The speed of light is a constant.

2. The speed of light is not subject to time dilation.

Note that both of these references refer to 'the speed of light' they do not refer to the measurement of the speed of light, because neither of these facts apply to the measurement of the speed of light; only the clock used to measure the speed of light is subject to time dilation.

Let us assume the existence of a space ship which would be equipped with instrumentation which could measure the speed of light, (this would include a cæsium clock), and with an accelerometer which could record changes in the velocity of the space ship through space.

This ship would be located at a Lagrange point; the force of gravity at that location could be determined, thereby eliminating the effect of gravitational time dilation.

The first step would be to measure, and record, the speed of light at this initial location. Assuming that the space ship was moving through space, the clock would be subject to time dilation resulting from the speed of the space ship through space, so the speed of light that we measured must be less than the absolute speed of light.

The objective of the experiment would be to reduce the speed of the space ship through space and thereby increase the measured speed of light - the slower the speed of the space ship through space, the less the time dilation and the greater the measured speed of light. If we could reduce the speed of our space ship through space to zero, the measured speed of light would be at its maximum, or absolute, value.

The experiment would start by establishing an $\mathrm{x}$-axis - its initial orientation would be irrelevant and change the velocity of our space ship along that axis. Initially it would not be known whether this change in the velocity had increased or decreased the velocity of the ship through space, but by re-measuring the speed of light this could be determined, because if the speed of the space ship through space had been reduced the time dilation the space ship experienced would also have decreased and the measured speed of light would have increased, and vice versa.

Using the measured speed of light as a guide, we could then continue to modify the velocity of our space ship through space along the $x$-axis until the measured speed of light was at its maximum value.

Next we would use our accelerometer to define a $y$-axis, perpendicular to the $x$-axis and follow the same procedure along the $y$-axis until again the measured speed of light was maximized. Finally we would establish a $z$-axis, perpendicular to the $x$-axis and $y$-axis and minimize our speed there also.

We would then have a location in space from which any change in speed in any direction would increase our speed through space and decrease the measured speed of light, and the resultant speed of light, measured at that location, would be the true absolute speed of light.

Comparing this number with our original measured speed of light would allow us to calculate the time dilation of earth and thus of other measurements of speed or velocity.

The readings on our accelerometer could be used to determine how we had modified our speed to reach zero, so we could determine the time dilation of objects that were not travelling at the same velocity as the original velocity of the space ship. 
Obviously the cost of the above experiment would not be trivial, but would it be greater than the cost of the CERN particle accelerator?

\section{References}

${ }^{1}$ Michelson, Albert A., Morley, Edward W.; (1887) "On the Relative Motion of the Earth and the Luminiferous Ether". American Journal of science. 34: 333-345

2 Einstein, Albert, "On the Electrodynamics of Moving Bodies", translated by W. Perrett and G.R. Jeffery, in the Principle of Relativity, New York: Fover and Methuen, 1952

${ }^{3}$ Self, Sydney B. "The Nature of Time - A $21^{\text {st }}$ Century View'. Journal of Advances in Physics, (2018) Volume 14, number 1.

${ }^{4}$ Self, Sydney B. "Measuring Velocity". Fundamental Journal of Modern Physics, Vol. 10, Issue 2 2017, Pages 161-164.

${ }^{5}$ Self, Sydney B. "A logical examination of the nature of time". Physics Essays 29, 2 (2016)

${ }^{6}$ Hafele, J.C., Keating, R.E., (July 14, 1972). "Around-the-World Atomic Clocks, Predicted Relativistic Time Gains". Science, 177

\section{Biography}

I was born in October, 1924. After $2 \frac{1 / 2}{2}$ years in the Army I graduated from M.I.T. in 1950 with a B.S. in Engineering Administration.

I received an M.B.A. from Northeastern University in1962.

I spent the next 40 years working in a variety of organizations designing, programming and installing Accounting Systems.

After I retired in 1992 I became interested in the subject of time and after reading a number of books on the subject I started writing articles, using logical analysis, about various aspects of time.

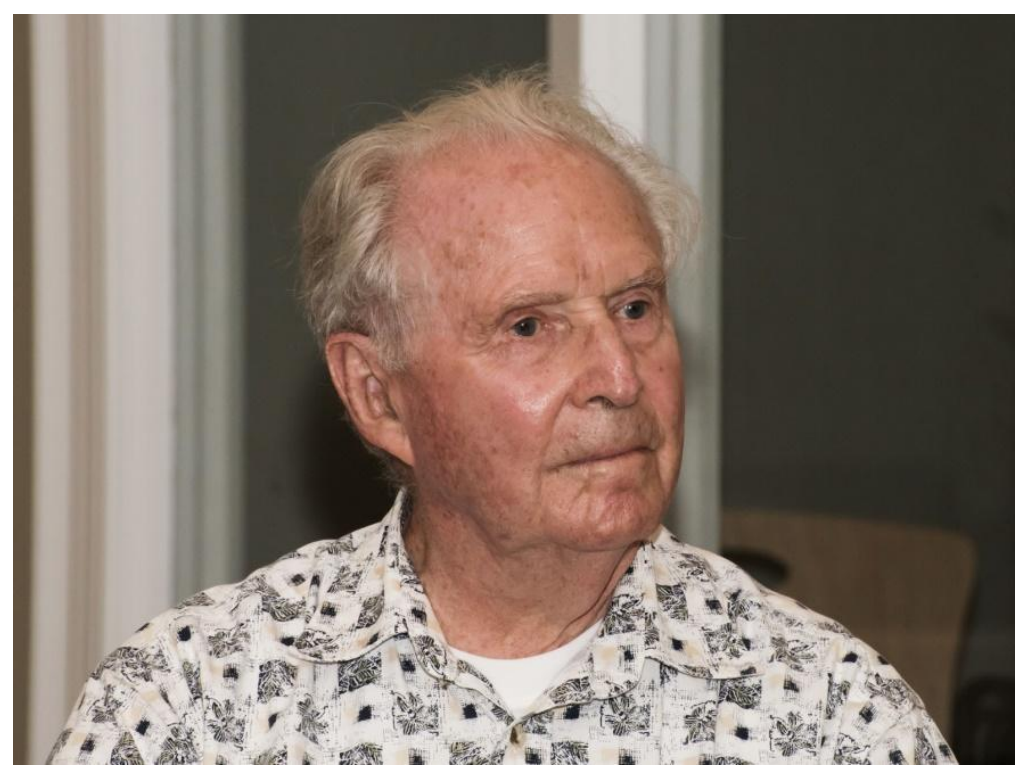

